\title{
Arsenic induced patterns of auto-grooming response over time in Macrobrachium lamarrei: a study on behavioural plasticity
}

Chayan Munshi ( $\nabla$ chayanbio@gmail.com )

Bengal Institute of Technology and Management

Deepyaman Das

Raiganj University

Partho Biswas

Visva Bharati University

Kosturi Sen

Visva Bharati University

Nisha Mondal

Visva Bharati University

Shreya Mukherjee

Visva Bharati University

Rajasree Bhowmick

Visva Bharati University

Piyali Kundu

Midnapore College

\section{Brief Communication}

Keywords: Repetitive grooming, Neurotoxicity, Behavioural toxicity, Stress, ASD

Posted Date: December 1st, 2020

DOl: https://doi.org/10.21203/rs.3.rs-113016/v1

License: (c) (1) This work is licensed under a Creative Commons Attribution 4.0 International License.

Read Full License 
1 Arsenic induced patterns of auto-grooming response over time in Macrobrachium 2 lamarrei: a study on behavioural plasticity

3

4 Chayan Munshi ${ }^{*}$, Deepyaman Das ${ }^{2}$, Partho Biswas ${ }^{3}$, Kosturi Sen ${ }^{3}$, Nisha Mondal ${ }^{4}$, Shreya $5 \quad$ Mukherjee $^{4}$, Rajasree Bhowmick ${ }^{3}$, Piyali Kundu ${ }^{5}$

6

$7 \quad{ }^{1}$ Bengal Institute of Technology and Management, Santiniketan, India

$8 \quad{ }^{2}$ Department of Microbiology, Raiganj University, Raiganj, 733134, India

$9 \quad{ }^{3}$ Department of Botany, Visva Bharati University, Santiniketan, 731235, India

$10{ }^{4}$ Department of Zoology, Visva Bharati University, Santiniketan, 731235, India

$11 \quad{ }^{5}$ Midnapore College, Medinipur, 721101, India

12

*Corresponding author email address: chayanbio@gmail.com

14

15

16

17

18

19

20

21

22

23

24

25 


\section{Abstract}

27 Behavioural toxicity is a very applicable area in the vast field of ecotoxicology where, assessment of the alteration of behaviour is an effective tool to evaluate neural activity in an organism. In our present study, we have shown that arsenic trioxide exposure can stimulate repetitive grooming activity in a freshwater prawn, Macrobrachium lamarrei; however, over time the organism can modulate the grooming performances to less. We predict that repetition in the grooming behaviour is a case of neurotoxicity by arsenic and induction of Autism Spectrum Disorder (ASD) within a short period of exposure time.

34

Keywords - Repetitive grooming, Neurotoxicity, Behavioural toxicity, Stress, ASD

36 


\section{Introduction}

Behavioural plasticity is an area of concern for many behavioural biologists. Research on the effect of pollutants and climate change on the behaviour of animals have taken an important place in the area of ethological research. Heavy metal contamination in the aquatic ecosystems is known to affect the normal neuronal activity in the organisms residing in that habitat, which eventually results in the induction of abnormal behavioural patterns. In the previous work by Munshi and Bhattacharya (2020) ${ }^{1}$, notable enhancement of auto-grooming (grooming) activity has been reported in Macrobrachium lamarrei (a freshwater prawn), due to a certain dose of non-lethal arsenic trioxide ( 24 hours of exposure). Arsenic is a well-known neurotoxicant ${ }^{2}$. In the present study, we have continued the study up to 72 hours of exposure time to evaluate the grooming response pattern over time. The aim of the study was to analyse, whether the organisms is effectively coping up with the contaminated environment and adjusting the time budget of grooming act or not. Behavioural toxicology is an emerging area in the field of ecotoxicology ${ }^{3}$. Analysis of the behavioural markers are very reliable tools in the area of neurobiological or neuroethological research. Normally, grooming behaviour in mammals has been studied as a behavioural marker of neuropsychiatric disorders ${ }^{4}$. In fact, repetitive grooming behaviour is an indication of autism spectrum disorders ${ }^{5}$. However, behaviour analysis in the area of environmental toxicology is a promising field in organismal research. Heavy metals are already given serious attention due to its adverse role in creating the neurological disorders ${ }^{6}$.

\section{Methodology}

Analysis of the alteration of auto-grooming behaviour in Macrobrachium lamarrei was done by assessing the grooming activity in the prawns in respect to spent time. Prawns were sampled from the natural ponds which is the natural habitat of these prawns. Collected prawns were maintained in glass aquarium and acclimatised in arsenic free, filtered water for 15 days. Food was provided ad libitum during the acclimatisation period. After this time, the grooming activity of 19 prawns were documented in the arsenic free water; this is our control group. Half of the prawn population used for the study, were gravid. Our study has sample size $n=19$. The same prawns were transferred to the experimental aquarium containing $1.7 \mathrm{ppm}$ concentration of arsenic trioxide. The grooming activity after 24 hours, 48 hours and 72 hours were documented; these are our three experimental groups. The behavioural documentation was done by considering a time frame of 300 seconds ( 5 minutes) after 24 hours, 48 hours and 72 
hours. During the whole process the temperature was maintained between $25-27^{\circ} \mathrm{C}$. All the graphical plots are plotted using, R4.0 and Python 3.8 programming language ${ }^{7,8}$ and Microsoft excel.

\section{Results and discussion}

From the previous work of Munshi and Bhattacharya $(2020)^{1}$, we have considered the fact that Macrobrachium lamarrei has a complex grooming pattern which is simplified into two major types: Anterior Grooming (AG) and Posterior Grooming (PG). We defined the terminologies, that were devised in our previous work ${ }^{1}$. In this article, we have discussed alterations in the magnitude of AG and PG in this species, over time. The magnitude of grooming (AG and PG) is measured in respect to time spent.

The prawns showed variability in AG and PG behavioural pattern. Compared to the control group, prawns in 24 hours of arsenic treatment had an elevation in the level of AG (1.2 folds), which was approximately same in 48 hours of arsenic treatment (1.15 folds). However, after 72 hours of treatment, the level of AG declined below the control, which is half of the level of AG in the control prawns. However, prawns after 24 hours of treatment had the increment of PG (17.6 folds) compared to the control. The level of PG after 48 hours and 72 hours of treatment was enhanced compared to the control but are roughly same (10.3 and 9.6 folds respectively). Prawns in the control group had greater levels of AG than PG (12.6 folds). However, after 24 hours of exposure, prawns showed greater level of PG than AG (1.15 folds). Again, prawns after 48 hours of exposure had greater level of AG than PG (1.4 folds). Lastly, the level of PG in prawns is again greater than AG after 72 hours of exposure (1.5 folds) (Figure $1 \mathrm{~A})$.

To summarise, from the patterns response of $A G$, it is clear that prawns showed increase in the level after 24 hours of treatment, however, there was a slight decrease in the level after 48 hours, which was still more than the control. However, after 72 hours, the level got decreased below the control. In the case of PG, prawns after 24 hours of treatment had high elevation of PG level, which eventually was reduced after 48 hours and 72 hours of arsenic exposure; however, the levels after 48 hours and 72 hours are more or less same, but which is still many times higher than the control. Comparing to the rise in the levels of the PG after 24 hours is notably higher than AG after 24 hours, which is sustained there after 48 hours. But in case of PG, after 48 hours, the decrease is noticeable, which is maintained there after 72 hours. After 72 hours, both AG and PG has declined, which was sharp in case of the AG (Figure 1B). 


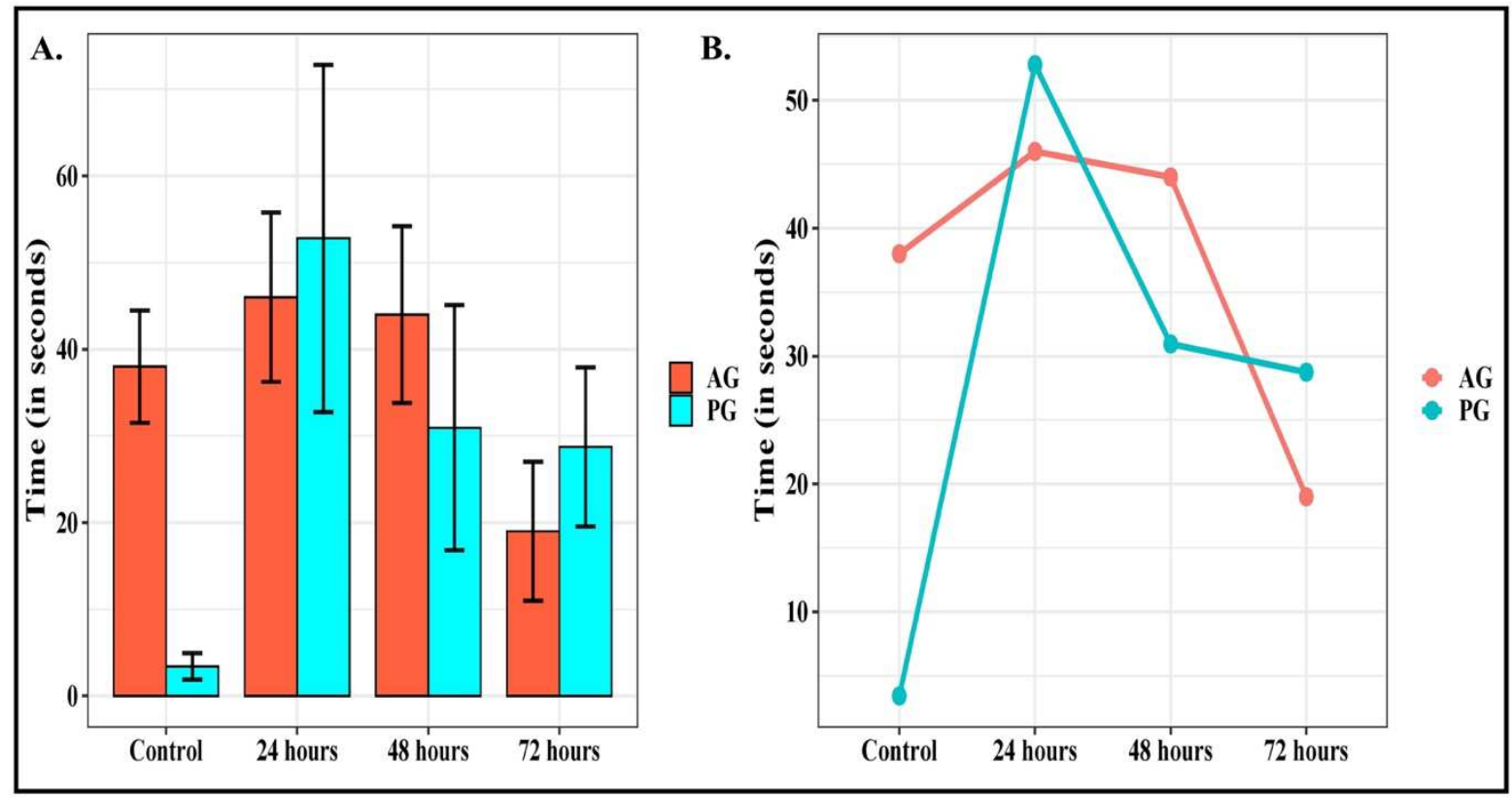

Figure 1: (A) Bar-plot and (B) Line diagram showing the alteration in Anterior Grooming (AG) and Posterior Grooming (PG) time budget in Macrobrachium lamarrei, over different arsenic trioxide exposure time ( 24 hours, 48 hours and 72 hours).

In the context of behavioural biology, all samples may not respond similarly to each condition and this forms the basis variability of a survey. The sample wise time budget for AG and PG shows myriad variability across groups thus indicating the randomness of the data (Figure 2a).

The grooming behaviour is divided into three clusters based on timing budget. Any behavioural change lasting not more than 100 seconds represent 'low' cluster followed by 101-200 seconds 'mid' cluster and greater than 200 seconds (up to 300 seconds) to be 'high' cluster. Prawns in the control group, have not crossed the low cluster for both AG and PG (100\%). In respect to the control, in the 24 hours post treatment, there was a decrease in the percentage of prawns in the low cluster, doing AG (from $100 \%$ to $84.2 \%$ ) but increase in the percentage of prawns in mid cluster (from 0 to $15.8 \%$ ). There were no prawns in the high cluster. There was also a decrease in the percentage of prawns in the low cluster, doing PG (from 100\% to $79.0 \%$ ) but increase in the percentage of prawns in both mid and high cluster, doing PG (0 to $10.5 \%$ for both clusters).

Compared to the control, for 48 hours post treatment group showed reduction in the percentage of prawns in the low cluster, doing AG (100\% to 89.5\%) but increase in the percentage of prawns in mid cluster ( 0 to $10.5 \%$ ). There were no prawns in the high cluster. Percentage of 
151

152

prawns doing PG is same as that of AG (100\% to $89.5 \%)$ but the percentage of prawns has increased in mid and high cluster, doing PG (almost same 0 to 5.3\% and 5.2\% respectively).

Linking to the control, 72 hours post treatment group demonstrated reduction in the percentage of prawns in low cluster, doing AG (100\% to $94.8 \%)$ but increase in the percentage of prawns in mid cluster ( 0 to $5.2 \%$ ). There were no prawns in high cluster. The same pattern there for PG also (Figure 2B).

To summarise, in low cluster, percentage of prawns was higher in doing AG than PG after 24 hours of exposure. However, after 48 and 72 hours of exposure, percentage of prawns doing AG and PG were same, which was greater than the percentage of prawns doing AG and PG after 24 hours. Among these experimental groups, the percentage of prawns for both AG and PG in low cluster were highest. In mid cluster, percentage of prawns was higher in AG than PG after 24 and 48 hours of exposure. However, percentage of prawns were same in doing AG and PG after 72 hours of exposure (Figure 2B.)

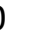

1

A.

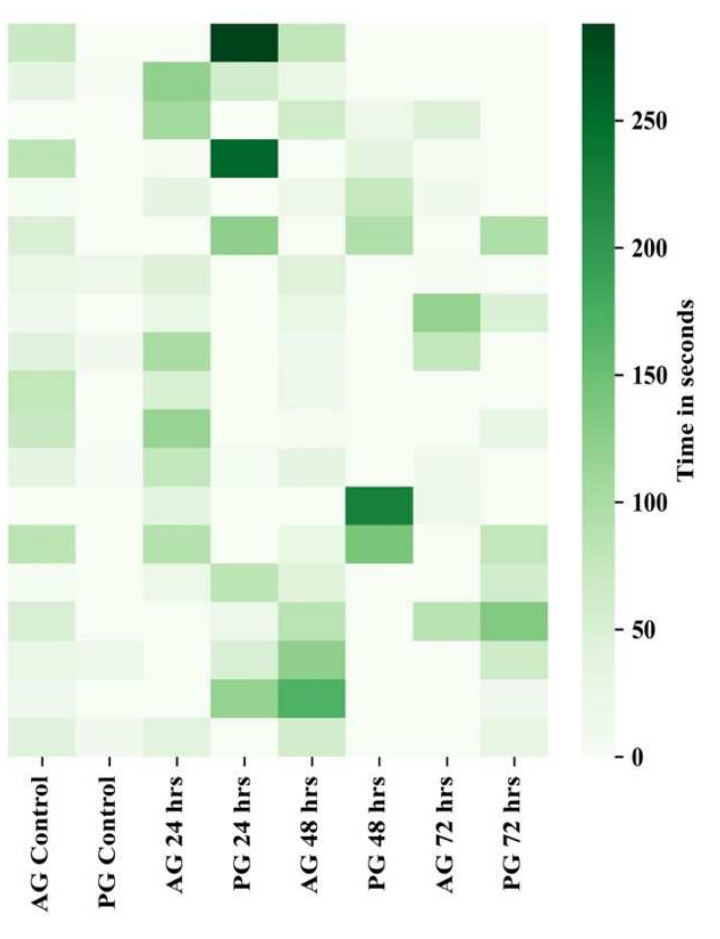

B.

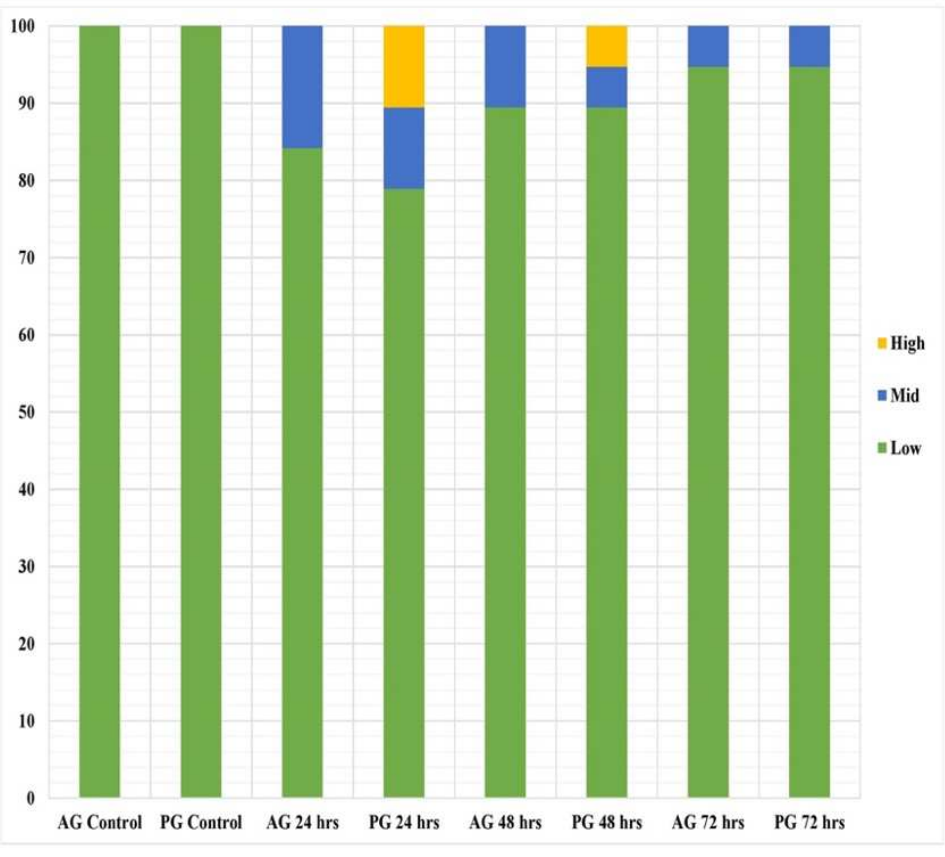


Figure 2: (A) Heatmap analysis (B) stacked graph representation to show the change in the number of Macrobrachium lamarrei, doing Anterior Grooming (AG) and Posterior Grooming $(\mathrm{PG})$ in different arsenic trioxide exposure time (24 hours, 48 hours and 72 hours). Hrs $=$ Hours.

\section{Conclusion}

Arsenic contamination in water is inducing the increase in the grooming activity in the freshwater prawn, Macrobrachium lamarrei which validates our previous work ${ }^{1}$. However, over time, analysis of the grooming performance elucidates that both AG and PG activities went at the highest point and eventually both went down over time. However, there was a contrasting results between AG and PG. The increase in the AG performances after 24 hours of exposure, led to decrease in the activity over time and eventually which declined below the control. But, in case of PG, we there was a drastic increase in the performance which also declined but remained notably high after 72 hours also compared to the control (Figure 1B).

$100 \%$ of the prawns showed low AG and PG performance in the control group. Only $10.5 \%$ of prawns after 24 hours of treatment showed high amount of PG, followed by $5.2 \%$ of prawns doing PG after 48 hours of PG. No prawns demonstrated high amount of AG after treatment. Exposure to arsenic, actually reduced the number of prawns in low cluster (from 100\%) and eventually the number has increased in the mid cluster; however, number of prawns performing AG is more than the number doing PG (except same in 72 hours post treatment) (Figure 2B). Initially the arsenic induced stress can stimulate enhanced in grooming activity which is an indication of initial shock. However, over time it can cope up with the stress and can eventually regulate the grooming performances. Though prawns do not go for excessive grooming but still arsenic showed the effect to enhance the grooming activity robustly. Repetitive behaviour is an indication of neurological disorders. In fact, repetitive grooming indicates a clear case of neurotoxicity and induction of Autism Spectrum Disorder (ASD) in a short time exposure to arsenic trioxide. 


\section{References}

195

196

197

198

199

200

201

202

203

204

205

206

207

208

209

210

211

212

213

214

1. Munshi, C. \& Bhattacharya, S. Behavioural toxicity of arsenic trioxide: alteration in auto-grooming behaviour of a freshwater prawn, Macrobrachium lamarrei. Austin J. Environ. Toxicol. 6, 1-5 (2020).

2. Sarkar, S., Mukherjee, S., Chattopadhyay, A. \& Bhattacharya, S. Low dose of arsenic trioxide triggers oxidative stress in zebrafish brain: Expression of antioxidant genes. Ecotoxicol. Environ. Saf. 107, 1-8 (2014).

3. Gerhardt, A. Aquatic behavioral ecotoxicology - Prospects and limitations. Hum. Ecol. Risk Assess. 13, 481-491 (2007).

4. Kalueff, A. V. et al. Neurobiology of rodent self-grooming and its value for translational neuroscience. Nat. Rev. Neurosci. 17, 45-59 (2016).

5. Blundell, J. et al. Neuroligin-1 deletion results in impaired spatial memory and increased repetitive behavior. J. Neurosci. 30, 2115-2129 (2010).

6. Gorini, F., Muratori, F. \& Morales, M. A. The Role of Heavy Metal Pollution in Neurobehavioral Disorders: a Focus on Autism. Rev. J. Autism Dev. Disord. 1, 354372 (2014).

7. R Core Team. R: A Language and Environment for Statistical Computing. (2020).

8. Hunter, J. D. Matplotlib: A 2D graphics environment. Comput. Sci. Eng. 9, 90-95 (2007). 
Figures
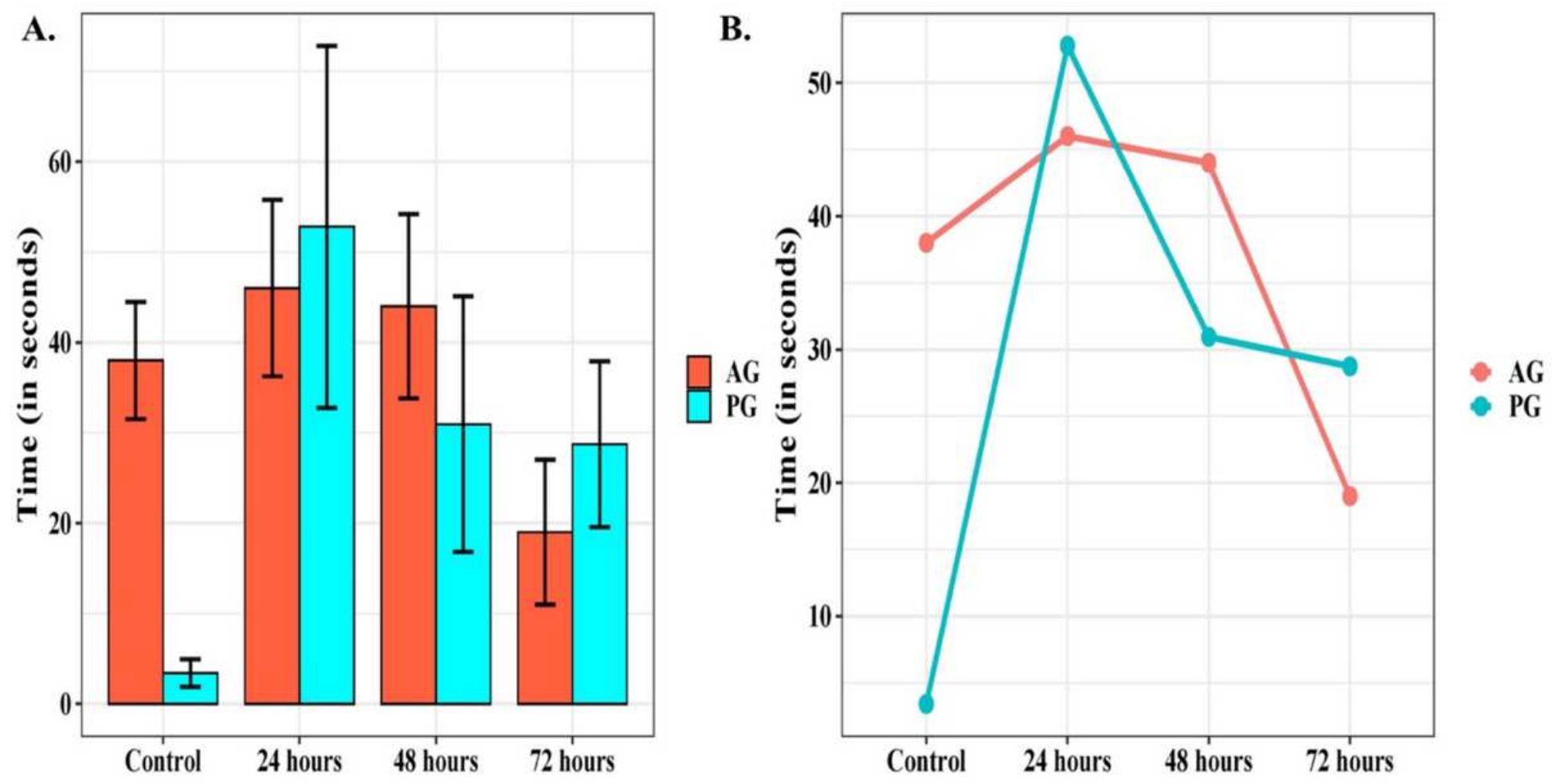

Figure 1

(A) Bar-plot and (B) Line diagram showing the alteration in Anterior Grooming (AG) and Posterior Grooming (PG) time budget in Macrobrachium lamarrei, over different arsenic trioxide exposure time (24 hours, 48 hours and 72 hours). 
A.

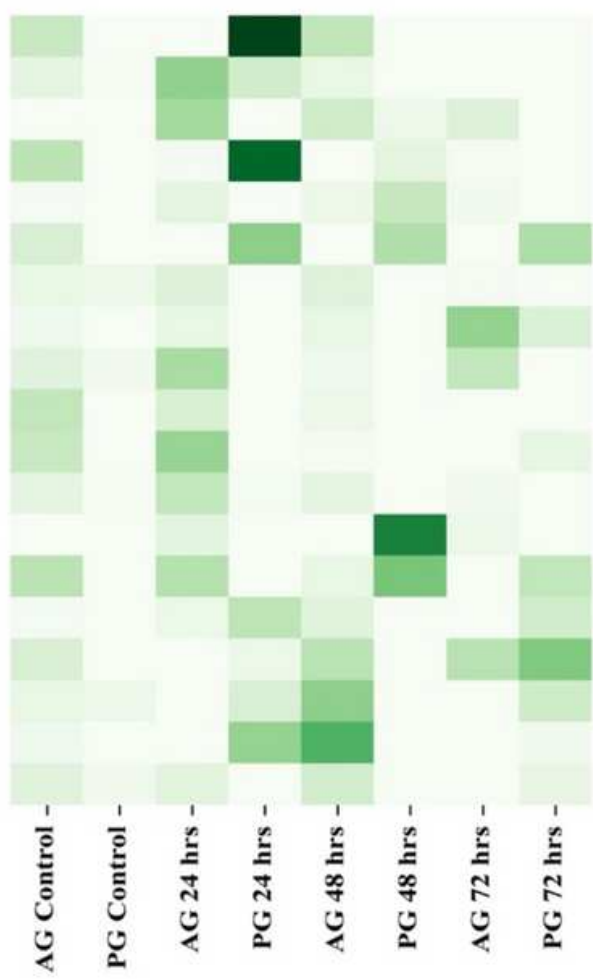

B.

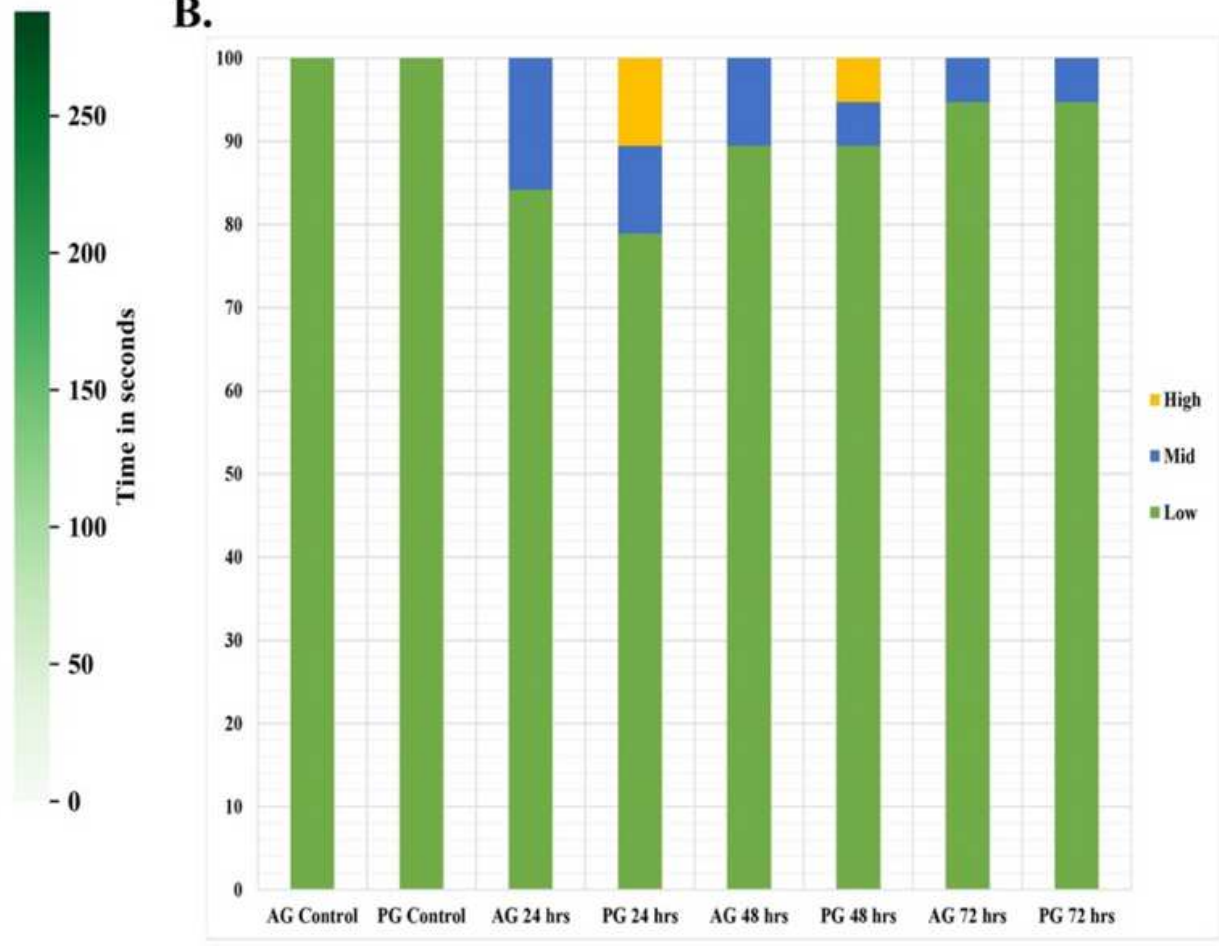

Figure 2

(A) Heatmap analysis (B) stacked graph representation to show the change in the number of Macrobrachium lamarrei, doing Anterior Grooming (AG) and Posterior Grooming (PG) in different arsenic trioxide exposure time (24 hours, 48 hours and 72 hours). Hrs $=$ Hours. 